Radar Signal Categorization using a Neural Network

James A. Anderson

Department of Cognitive and Linguistic Sciences

Box 1978

Brown University, Providence, RI 02912

and

Michael T. Gately, P. Andrew Penz, and Dean R. Collins

Central Research Laboratories, Texas Instruments

Dallas, Texas 75265

Accepted for Publication, IEEE Proceedings

To appear, Augus $\bar{t}, 1990$

(c) IEEE

This research was initially supported by Texas Instruments, the Office of Naval Research (Contract N00014-86-K-0600 to J.A.) and the National Science Foundation (Grant BNS-85-18675 to J.A.). Currently, this research is supported by the Avionics Laboratory, Wright Research and Development Center, Aeronautical Systems Division [Contract F33615-87-C1454]. 


\title{
Radar Signal Categorization Using a Neural Network
}

\begin{abstract}
Neural networks were used to analyze a complex simulated radar environment which contains noisy radar pulses generated by many different emitters. The neural network used is an energy minimizing network (the BSB model) which forms energy minima -attractors in the network dynamical system -- based on learned input data. The system first determines how many emitters are present (the deinterleaving problem). Pulses from individual simulated emitters give rise to separate stable attractors in the network. Once individual emitters are characterized, it is possible to make tentative identifications of them based on their observed parameters. As a test of this idea, a neural network was used to form a small data base that potentially could make enitter identifications.
\end{abstract}

We have used neural networks to cluster, characterize and identify radar signals from different emitters. The approach assumes the ability to monitor a region of the microwave spectrum and to detect and measure properties of received radar pulses. The microwave environment is assumed to be complex, so there are pulses from a number of different emitters present, and pulses from the same emitter are noisy or their properties are not measured with great accuracy.

For several practical applications, it is important to be able to tell quickly, first, how many emitters are present and, second, what their properties are. In other words time average prototypes must be derived from time dependent data without a tutor. Finally the system must tentatively identify the prototypes as members of previously seen classes of emitter.

Stages of Processing. We accomplish this task in several stages. Figure 1 shows a block diagram of the resulting system, which contains several neural networks. The system as a whole is referred to as the Adaptive Network Sensor Processor (ANSP).

Figure 1 About Here

In the block diagram given in Figure 1, the first block is a feature extractor. We start by assuming a microwave radar receiver of some sophistication at the input to the system. This receiver is capable of processing each pulse into feature values, i.e. azimuth, elevation, signal to noise ratio (normalized intensity), frequency, and pulse width. This data is then listed in a pulse buffer and tagged with time of arrival of the pulse. In a complex radar environment, hundreds or thousands of pulses can arrive in fractions of seconds, so there is no lack of data. The problem, as in many data rich environments, is making sense of it. 
The second block in Figure 1 is the deinterleaver which clusters incoming radar pulses into groups, each group formed by pulses from a single emitter. A number of pulses are observed, and a neural network computes, off line, how many emitters are present, based on the sample, and estimates their properties. That is, it solves the so-called deinterleaving problem by identifying pulses as being produced by a particular emitter. This block also produces and passes forward measures of the each cluster's azimuth, elevation, SNR, frequency and pulse width.

The third block, the pulse pattern extractor, uses the deinterleaved information to compute the pulse repetition pattern of an emitter by using the times of arrival for the pulses that are contained in a given cluster. This information will be used for emitter classification.

The fourth block, the tracker, acts as a long term memory for the clusters found in the second block, storing the average azimuth, elevation, SNR, frequency, and pulse width. Since the diagram in Figure 1 is organized via initial computational functionality, the tracking module follows the deinterleaver so as to store its outputs. In an operationally organized diagram, the tracker is the first block to receive pulse data from the feature extractor. It must identify most of the pulses in real time as previously learned by the deinterleaver module and only pass a small number of unknown pulses back to the deinterleaver module for further learning. The tracker also updates the cluster averages. Their properties can change with time because of emitter or receiver motion, for example.

The fourth and fifth blocks, the tracker and the classifier operate as a unit to classify the observed emitters, based on information stored in a data base of emitter types. Intrinsic emitter properties stored in these blocks are frequency, pulse width and pulse repetition pattern.

The most important question for the ANSP to answer is what the emitters might be and what can they do. That is, "who is looking at me, should I be concerned, and should I (or can I) do something about it?"

Emitter clustering. Most of the initial theoretical and simulation effort in this project has been focused on the deinterleaving problem. This is because the ANSP is being asked to form a conception of the emitter environment from the data itself. A teacher does not exist for most interesting situations.

In the simplest case, each emitter emits with constant properties, i.e. no noise is present. Then, determining how many emitters were present would be trivial: simply count the number of unique pulses via a look up table. Unfortunately, data is often moderately nolsy because of receiver, environmental and emitter variability, and, sometimes, because of the frequent change of one or another emitter property at the emitter. Therefore, simple identity checks will not work. It is these later cases which this paper will address.

Many neural networks are supervised algorithms, that is, they are trained by seeing correctly classified examples of training data and, when new data is presented will identify it according to their past experience. Emitter identification does not fall into this category because the correct answers are not known ahead of time. That, after all, is the purpose of this system. The basic problem of a self-organizing clustering system has many historical precedents in cognitive science. For example, William James, in a quotation well known to developmental psychologists, wrote around 1890 , 
..the numerous inpouring currents of the baby bring to his consciousness ... one big blooming buzzing Confusion. That Confusion is the baby's universe; and the universe of all of us is still to a great extent such a Confusion, potentially resolvable, and demanding to be resolved, but not yet actually resolved into parts.

William James (1890, p.29)

We now know that the new born baby is a very competent organism, and the outlines of adult perceptual preprocessing are already in place. The baby is designed to hear human speech in the appropriate way and to see a world like ours: that is, a baby is tuned to the environment in which he will live. The same is true of the ANSP, which must process pulses which will have feature values that fall within certain parameter ranges. That is, an effective feature analysis has been done for us by the receiver designer, and we do not have to organize a system from zero. This means that we can use a less general approach than we might have to in a less constrained problem. The result of both evolution and good engineering design is to build so much structure into the system that a problem, very difficult in its general form, becomes quite tractable.

At this point, neural networks are familiar to many. Introductions are available, for example, Mcclelland and Rumelhart, 1986; Rumelhart and McClelland, 1986; Hinton and Anderson, 1989; Anderson and Rosenfeld, 1988.

The Linear Associator. Let us begin our discussion of the network we shall use

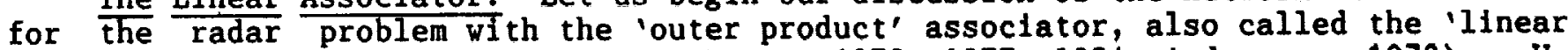
associator,' as a starting point. (Kohonen, 1972, 1977, 1984; Anderson, 1972). We assume a single computing unit, a simple model neuron, acts as a linear summer of its inputs. There are many such computing units. The set of activities of a group of units is the system state vector. Our notation has matrices represented by capital letters (A), vectors by lower case letters $(f, g)$, and the elements of vectors as $f(i)$ or $g(j)$. A vector from a set of vectors is subscripted, for example, $f_{1}, f_{2} \cdots$.

The ith unit in a set of units will display activity $g(i)$ when a pattern $f(j)$ is presented to its inputs, according to the rule,

$$
g(i)=\sum_{j} A(i, j) f(j) \text {. }
$$

where $A(i, j)$ are the connections between the $i$ th unit in an output set of units and the $j$ th unit in an input set. We can then can write the output pattern, $g$, as the matrix multiplication

$$
\boldsymbol{g}=\mathrm{A} \mathbf{f} \text {. }
$$

During learning, the connection strengths are modified according to a generalized Hebb rule, that is, the change in an element of $A, \delta A(i, f)$, is given by 


$$
\delta A(i, j) \propto \underset{k}{f(j)} \underset{k}{g(i)}
$$

where $f_{k}$ and $g_{k}$ are vectors associated with the $k$ th learning example.

Then we can write the matrix $A$ as a sum of outer products,

$$
A=\eta_{k=1}^{n} \underset{k}{g} \underbrace{T}
$$

where $n$ is a learning constant.

Prototype Formation The linear model forms prototypes as part of the storage process, a property we will draw on. Suppose a category contains many similar items associated with the same response. Consider a set of correlated vectors, $\left\{f_{k}\right\}$, with mean $p$.

$$
\mathrm{f}_{\mathrm{k}}=\mathrm{p}+\mathrm{d}
$$

The final connectivity matrix will be

$$
\begin{aligned}
A & =\underset{k=1}{n \mathcal{L} g} \underset{k}{\mathrm{~T}} \\
& =n g\left(n p^{T}+\sum_{k=1}^{n} d_{k}^{T}\right)
\end{aligned}
$$

If the sum of the $d_{k}$ is small, the connectivity matrix is approximated by

$$
A=m g p^{T} \text {. }
$$

The system behaves as if it had repeatedly learned only one pattern, $p$, and responds best to it, even though $p$, in fact, may never have been learned.

Concept forming systems. Knapp and Anderson (1984) applied this model directly to the formation of simple psychological 'concepts' formed of nine randomly placed dots. A 'concept' in cognitive science describes the common and important situation where a number of different objects are classed together by some rule or similarity relationship. Much of the power of language, for example, arises from the ability to see that physically different objects are really 'the same' and can be named and responded to in a similar fashion, for example, tables or lions. A great deal of experimentation and theory in cognitive science concerns itself with concept formation and use.

There are two related but distinct ways of explaining simple concepts in neural network models. First, there are prototype forming systems, which often involve taking a kind of average during the act of storage, and, second, there are models which explain concepts as related to attractors in a dynamical system. In the radar ANSP system to be described we use both ideas: we want to construct a system where 
the average of a category becomes the attractor in a dynamical system, and an attractor and its surrounding basin represent an individual emitter. (For a further discussion of concept formation in simple neural networks, see Knapp and Anderson, 1984; Anderson, 1983, and Anderson and Murphy, 1986).

Error Correction. By using an error correcting technique, the Widrow-Hoff procedure, we can force the simple associative system to give us more accurate associations. Let us assume we are working with an autoassociative system. Suppose information is represented by associated vectors $f_{1} \Rightarrow f_{1}, f_{2} \Rightarrow f_{2} \ldots$. A vector, $f_{k}$, is selected at random. Then the matrix, $A$, is incremented according to the rule

$$
\Delta A=n(f-A f) f_{k}^{T}
$$

where $\triangle A$ is the change in the matrix $A$. In the radar application, there is no 'correct answer' in the general sense of a supervised algorithm. However every input pattern can be its own 'teacher' in the error correction algorithm in that the network will try to better reconstruct that particular input pattern. The goal of learning a set of stimuli (f) is to have the system behave as

$$
A \underset{k}{f}=\mathbf{f}
$$

The error correcting learning rule will approximate this result with a least mean squares approximation, hence the alternative name for the Widrow-Hoff rule: the LMS (least mean squares) algorithm. The autoassociative system combined with error correction, when working perfectly, is forcing the system to develop a particular set of eigenvectors with eigenvalue 1 .

The eigenvectors of the connection matrix are also of interest when simple Hebbian learning is used in an autoassociative system. Then, the simple outer product associator has the form

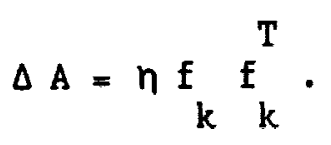

There is now an obvious connection between the eigenvectors of the resulting outer product connectivity matrix and the principal components of statistics, because the form of this matrix is the covariance matrix. In fact, there is growing evidence that many neural networks are doing something like principal component analyis. (See, for example, Baldi and Hornik, 1989 and Cottrell, Munro and Zipser, 1988).

BSB: A. Dynamical System. We shall use for radar clustering a non-linear model that takes the basic linear associator, uses error correction to construct the connection matrix, and uses units containing a simple limiting non-linearity. Consider an autoassociative feedback system, where the vector output from the matrix is fed back into the input. Because feedback systems can become unstable, we incorporate a simple limiting non-linearity to prevent unit activity from getting too large or too small. Let $f[i]$ be the current state vector describing the system. $f[0]$ is the vector at step 0 . At the $i+1 s t$ step, $f[i+1]$, the next state vector, is given by the iterative equation, 


$$
f[i+1]=\operatorname{LIMIT}[\alpha \mathrm{Af}[\mathrm{i}]+\gamma \mathrm{f}[\mathrm{i}]+\delta \mathrm{f}[0]]
$$

We stabilize the system by bounding the element activities within limits.

The first term, $\alpha A f[i]$, passes the current system state through the matrix and adds information reconstructed from the autoassociative cross connections. The second term, $r f[i]$, causes the current state to decay slightly. This term has the qualitative effect of causing errors to eventually decay to zero as long as $r$ is less than 1. The third term, $\delta f[0]$, can keep the initial information constantly present and has the effect of limiting the flexibility of the possible states of the dynamical system since some vector elements are strongly biased by the initial input.

Once the element values for $f[i+1]$ are calculated, the element values are 'limited', that is, not allowed to be greater than a positive limit or less than a negative iimit. This is a particularly simple form of the sigmoidal nonlinearity assumed by most neural network model. The limiting process contains the state vector within a set of limits, and we have previously called this model the 'brain state in a box' or BSB model. (Anderson, Silverstein, Ritz, and Jones, 1977; Anderson and Mozer, 1981) The system is in a positive feedback loop but is amplitude limited. After many iterations, the system state becomes stable and will not change: these points are attractors in the dynamical system described by the BSB equation. This final state will be the output of the system. In the fully connected case with a symmetric connection matrix the dynamics of the BSB system can be shown to be minimizing an energy function. The location of the attractors is controlled by the learning algortthm. (Hopfield, 1982; Golden, 1986). Aspects of the dynamics of this system are related to the 'power' method of eigenvector extraction, since repeated iteration will leada to activity dominated by the eigenvectors with the largest postive eigenvalues. The signal processing abilities of such a network occur because eigenvectors arising from learning uncorrelated noise will tend to have small eigenvalues, while signal related eigenvectors will be large, will be enhanced by feedback, and will dominate the system state after a number of iterations.

We might confecture that a category or a concept derived from many notsy examples would become identified with an attractor associated with a region in state space and that all examples of the concept would map into the point attractor. This is the behavior we want for radar pulse clustering.

Neural Network Clustering Algorithms. We know there will be many radar pulses, but we do not know the detailed descriptions of each emitter invoved. We want to develop the structure of the microwave environment, based on input information. A number of models have been proposed for this type of task, including various competitive learning algorithms (Rumelhart and Zipser, 1986; Carpenter and Grossberg, 1987).

Each pulse is different because of noise, but there are only a small number of emitters present relative to the number of pulses. We take the input data representing each pulse and form a state vector with it. A sample of several hundred pulses are stored in a 'pulse buffer.' We take a pulse at random and learn it, using the Widrow-Hoff error correcting algorithm with a small learning constant. Since there is no teacher, the desired output is assumed to be the input pulse data.

Learning rules for this class of dynamical system, Hebbian learning in general, (Hopfield, 1982) and the Widrow-Hoff rule in particular, are effective at idigging holes in the energy landscape' so they fall where the vectors that are learned are. That is, the final low energy attractor states of the dynamical system when BSB 
dynamics are applied will tend to lie near or on stored information. Suppose we learn each pulse as it comes in, using Widrow Hoff error correction, but with a small learning constant. Metaphorically, we 'dig a little hole' at the location of the pulse. But each pulse is different. So, after a while, we have dug a hole for each pulse, and if the state vectors coding the pulses from a single emitter are not too far apart in state space, we have formed an at tractor that contains all the pulses from a single emitter, as well as new pulses from the same emitter. Figure 2 presents a (somewhat fanciful) picture of the behavior that we hope to obtain, where many nearby data points combine to give a single broad network energy minimum that contains them all.

Figure 2 about here

We can see why this behavior will occur from an informal argument. Call the average emitter state vector of a particular emitter $p$. Then, every observed pulse, $\mathrm{f}_{\mathrm{k}}$, will be

$$
\mathrm{f}_{\mathrm{k}}=\mathrm{p}+\mathrm{d}, \mathrm{k}
$$

where $d_{k}$ is a distortion, which will be assumed to be different for every individual pulse, $k_{\text {that }} i s$, different $d_{k}$ are uncorrelated, and are relatively small compared to p. With a small learning constant, and with the connection matrix A starting from zero, the magnitude of the output vector, Af, will also be small after only a few pulses are learned. This means that the error vector will point outward, toward $f_{k}$, that is, toward $p+d_{k}$, as shown in Figure 3 .

Figure 3 about here

Early in the learning process with a small learning constant for a particular cluster, the error vectors (input minus output) all will point toward the cluster of input pulses. Widrow Hoff learning can be described as using a simple associator to learn the error vector. Since every $d_{k}$ is different and uncorrelated, the error vectors from different pulses will have the average direction of $p$. The matrix will act as if it is repeatedly learning $p$, the average of the vectors. It is easy to show that if the centers of different emitter clusters are spaced far apart, in particular, if the cluster centers are orthogonal, then $p$ will be close to an eigenvector of $\mathrm{A}$. In more interesting and difficult cases, where clusters are close together or the data is very noisy, it is necessary to resort to numerical simulation to see how well the network works in practice. As we hope to show, this technique does work quite well.

After the matrix has learned so many pulses that the input and output vectors are of comparable magnitude, the output of the matrix when $p+d_{k}$ is presented will be near p. (See Figure 4) Then, 
Over a number of learned examples,

$$
\begin{aligned}
\text { total error } & \simeq \Sigma\left(\underset{k}{p+d_{k}}-A(p+d)\right. \\
& \simeq \Sigma\left(d_{k}-A d \underset{k}{A}\right)
\end{aligned}
$$

The maximum values of the eigenvalues of $A$ are 1 or below, the d's are uncorrelated, and this error term will average to zero.

Figure 4 about here

However, as the system learns more and more random noise, the average magnitude of the error vector will tend to get longer and longer, as the eigenvalues of $A$ related to the noise become larger. Note that system learning never stops because there is always an error vector to be learned, which is a function of the intrinsic noise in the system. Therefore, there is a 'senility' mechanism found in this class of neural networks. For example, the covariance matrix of independent, identically distributed Gaussian noise added to each element is proportional to the identity matrix, then every vector becomes an eigenvector with the same elgenvalue, and this matrix is the matrix toward which $A$ will evolve, if it continues to learn random noise indefinitely. When the BSB dynamics are applied to matrices resulting from learning very large numbers of noisy pulses, the attractor basins become fragmented, so that the clusters break up. However, the period of stable cluster formation is very long and it is easy to avoid cluster breakup in practice. (Anderson, 1987)

In BSB clustering the desired output is a particular stable state. Ideally, all pulses from one emitter will be attracted to that final state. Therefore a simple identity check is now sufficient to check for clusters. This check is performed by resubmitting the original noisy pulses to the network that has learned them and forming a list of the stable states that result. The list is then compared with itself to find which pulses came from the same emitter. For example, a symbol could be associated with the pulses from the same final state, i.e. the pulses have been deinterleaved or identified.

Once the emitters have been identified, the average characteristics of the features describing the pulse (frequency, pulse width and pulse repetition pattern) can be computed. These features are used to classify the emitters with respect to known emitter types in order to 'understand' the microwave environment. A two stage system, which first clusters and then counts clusters is easy to implement, and, practically, allows convenient 'hooks' to use traditional digital techniques in conjunction with the neural networks.

Stimulus Coding and Representation. The fundamental represention assumption of almos all neural networks is that information is carried by the pattern or set of activities of many neurons in a group of neurons. This set of activities carries the 
meaning of whatever the nervous system is doing and these sets of activities are represented as state vectors. The conversion of input data into a state vector, that is, the representation of the data in the network, is the single most important engineering problem faced in network design. In our opinion, choice of a good input and output representation is usually more important for the ultimate success of the system than the choice of a particular network algorithm or learning rule.

We now suggest an explicit representation of the radar data. From the radar receiver, we have a number of continuous valued features to represent: frequency, elevation, azimuth, pulse width, and signal strength. Our approach is to code continuous information as locations on a topographic map, 1.e. a bar graph or a moving meter pointer. We represent each continuous parameter value by location of block of activation on a linear set of elements. Increase in a parameter value moves the block of activity to the right, say, and a decrease, moves the activity to the left. We have used a more complex topographic representation in several other contexts, with success. (Sereno, 1989; Rossen, 1989; Viscuso, Anderson, and Spoehr, 1989).

We represent the block/bar of activity value with a block (three or four) " $="$, equal, symbols placed in a region of ".", period, symbols. Single characters are coded by eight bit ASCII bytes. The ASCII $1^{\prime}$ 's and $0^{\prime} s$ are further transformed to +1 's and -1 's, so that the magnitude of any feature vector is the same regardless of the feature value. Input vectors are therefore purely binary. On recali, if the vector elements coding a character do not rise above a threshold size, the system is not 'sure' of the output. Then that character is represented as the underline, "-", character. Being 'not sure' can be valuable information relative to the confideñce of a particular output state relative to an input. Related work has developed a more numeric, topographic representation for this task, called a 'closeness code' (Penz, 1987) which has also been successfully used for clustering of simulated radar data.

Neural networks can incorporate new information about the signal and make good use of it. This is one version of what is called the data fusion or sensor fusion problem. To code the various radar features, we simply concatenate the topographic vectors of individual feature into a single long state vector. Bars in different fields code the different quantities. Figure 5 shows these fields.

Figure 5 about here

Below we will gradually add information to the same network to show the utility of this fusion methodology. The conjecture is is that adding more information about the pulse will produce more accurate clustering. Note that we can insert 'symbolic' information (say word identifications or other appropriate information) in the state vector as character strings, forming a hybrid code. For instance the state vector can contain almost unprocessed spectral data together with the symbolic bar graph data combined with character strings representing symbols at the same time.

A Demonstration. For the simulations of the radar problem that we describe next, we used a BSB system with the following properties. The system used 480 units, representing 60 characters. Connectivity was $25 \%$, that is, each element was connected at random to 120 others. There were a total of 10 simulated emitters with considerable added intrinisic noise. A pulse buffer of 510 different pulses was used for learning and, after learning, 100 new pulses, 10 from each emitter were used to 
test the system. There were about 2000 total learning trials, about that is, about four presentations per example. Parameter values were $\alpha=0.5, \gamma=0.9$ and $\delta=0$. The limits for thresholding were +2 and -2 . None of these parameters were critical, in that moderate variations of the parameters had little effect on the resulting classifications of the network.

Suppose we simply learn frequency information. Figure 6 shows the total number of attractors formed when ten new examples of each of ten emitters were passed through the BSB dynamics, using the matrix formed from learning the pulses in the pulse buffer. In a system that clustered perfectly, exactly 10 final states would exist, one different final state for each of the ten emitters. However, with only frequency information learned, all the 100 different inputs mapped into only two at tractors.

Figure 6 about here

Figure 6 and others like it below are graphical indications of the similarity between recalled clusters or states with computational energy minima. The states shown in the figures are ordered via a priori knowledge of the emitters, although this information was obviously not given to the network. One can visually interpret the outputs for equality of two emitters (lumping of different emitters) or separation of outputs for a single emitter (splitting of the same emitter) in the outputs. This display method is for the reader's benefit. The ANSP system determines the number and state vector of separate minima by a dot product search of the entire output list, as discussed above. Position of the bar of ' $=$ 's codes the frequency in the frequency field which is the only field learned in this example.

Let us now give the system additional information about pulse azimuth and elevation. Clustering performance improves markedly, as shown in Figure 7 . We get nine different attractors. There is still uncertainty in the system, however, since few corners are fully saturated, as indicated by the underline symbols on the corners of some bar's. States 1 and 3 are in the same attractor, an example of incorrect 'lumping' as a result of insufficient information. Two other final states (8 and 9) are very close to each other in Hamming distance.

Figure 7 about here

Let us assume that future advances in recelvers will allow a quick estimation of the microstructure of each radar pulse. We have used, as shown in Figure 8 , a coding which is a crude graphical version of a Fourier anlysis of an individual pulse, with the center frequency located at the middle of the field. Emitter pulse spectra were assigned arbitrarily. 
Figure 8 about here

Note that the spectral information can be included in the state vector in only slightly processed form: we have included almost a caricature of the actual spectrum.

Addition of spectral information improved performance somewhat. There were nine distinct attractors, though still many unsaturated states. Two emitters were still 'lumped', 8 and 9 . Figure 9 shows the results.

Figure 9 about here

Suppose we add information about pulse width to azimuth, elevation, and frequency. The simulated pulse width information is very poor. It actually degrades performance, though it does allow separation of a couple of nearby emitters. The results are given in Figure 10.

Figure 10 about here

The reason pulse width data is of poor quality and hurts discrimination is because of a common artifact due to the way that pulse width is measured. When two pulses occur close together in time a very long pulse width is measured by the receiver circuitry. This can give rise in unfavorable cases to a spurious bimodal distribution of pulsewidths for a single emitter. Therefore, a single emitter seems to have some short pulse widths and some very long pulse widths and this can split the category. Bimodal distributions of an emitter parameter, when the peaks are widely separated, is a hard problem for any clustering algorithm. A couple of difficult discriminations in this simulation, however, are aided by the additional data.

We now combine all this information about pulse properties together. None of the subsets of information could perfectly cluster the emitters. Pulse width, in particular, actually hurt performance. Figure 11 shows that, after learning, using all the information, we now get ten well separated attractors, i.e. the correct number of emitters relative to the data set. The conclusion is that the additional information, even if it was noisy, could be used effectively. Poor information could be combined with other poor information to give good results. 
Figure 11 about here

Processing After Deinterleaving. Having used the ANSP system to deinterleave and cluster data, we also have a way of producing an accurate picture of each emitter. We now have an estimate of the frequency and pulse width and can derlve other emitter properties (Penz et." al., 1989), for example, the emitter pulse repetition pattern. One method to learn this pattern is to learn pulse repetition interval (PRI) pairs autoassociatively. Another is to autocorrelate the PRI's of a string. This technique probably provides more information than any other for characterizing emitters, because the resulting correlation functions are very useful for characterizing a particular emitter type.

Classification Problem and Neural Network Data Bases. The next task is to classify the observed emitters based on our previous experience with emitters of various types. We continue with the neural network approach because of the ability of networks to incorporate a great deal of information from different sensors, their ability to generalize (i.e. 'guess') based on noisy or incomplete information, and their ability to handle ambiguity. Known disadvantages of neural networks used as data bases are their slow computation using traditional computer architectures, erroneous generalizations (i.e. 'bad guesses'), their unpredictability, and the difficulty of adding new information to them, which may require time consuming relearning.

Information, in traditional expert systems, is often represented as collections of atomic facts, relating pairs or small sets of items together. Expert systems often assume 'IF ( $x$ ) THEN (y)' kinds of information representation. For example, such a rule in radar might look like:

$$
\begin{aligned}
& \text { IF (Frequency is } 10 \mathrm{gHz} \text { ) } \\
& \text { AND (Pulse Width is } 1 \mathrm{microsecond)} \\
& \text { AND (PRI is constant at } 1 \mathrm{kHz} \text { ) }
\end{aligned}
$$

THEN (Emitter is a Klingon air traffic control radar).

Problems with this approach are that rules usually have many exceptions, data may be erroneous or noisy, and emitter parameters may be changed because of local conditions. Expert systems may be exceptionally prone to confusion when emitter properties change because of the rigidity of their data representation. Neural networks allow a different strategy: Always try to use as much information as you have, because, in most cases, the more information you have, the better performance will be.

As William James commented in the nineteenth century,

... the more other facts a fact is associated with in the mind, the better posession of it our memory retains. Each of its associates becomes a hook to which it hangs, a means to fish it up by when sunk beneath the surface. Together, they form a network of attachments by which it is woven into the entire 
Perhaps, as William James suggests, information is best represented as large sets of correlated information. We could represent this in a neural network by a large, multimodal state vector. Each state vector contains a large number of 'atomic facts' together with their cross correlations. Our clustering demonstration showed that more information could be added and used efficiently and that identification depends on a cluster of information co-occuring. (See Anderson, 1986 for further discussion of neural network data bases of this type.)

Ultimately, we would like a system that would tentatively identify emitters based on measured properties and previously known information. Since we know, in operation, that parameters can and often do change, we can never be sure of the answers.

As a specific important example, radar systems can shift parameters in ways consistent with their physical design, that is, waveguide sizes, power supply size, and so on, for a number of reasons, for example, weather conditions. If an emitter is characterized by only one parameter, and that parameter is changed, then identification becomes very unlikely. Therefore, accuracy of measurement of a particular parameter may not be as useful for classification as one might expect. However, using a whole set of co-occuring properties, each at low precision, may prove a much more efficient strategy for identification. For further discussion of how humans often seem to use such a strategy in perception, consult George Miller's classic 1956 paper, "The magic number seven, plus or minus two."

Classification Problem for Shifted Emitters. Our first neural net classification simulation is specifically designed to study sensitivity to shifts in parameters. Two data sets were generated. One set has 'normal' emitter properties and the other set had all the emitter properties changed about 10 percent. The two sets each contained about 500 data points. The names used are totally arbitrary. The state vector was constructed of a name string (the first 10 characters) and bar codes for frequency, pulse width, and pulse repetition interval. For the classification function, the position of "+" symbols indicates the feature magnitude while the blank symbol fills the rest of the feature field. Again the "_" symbol indicates an undecided node.

Figures 12 and 13 show the resulting attractor interpretations. Figure 12 shows the vectors to be learned autoassociatively by the BSB model. The first field is the emitter name. The last three fields represent the numerical information produced by the deinterleaver and pulse repetition interval modules. An input consists of leaving the identification blank and filling in the analog information for the emitter which one wants an identification. The autoassocative connections fill in the missing identification information.

Figure 12 shows the identifications produced when the normal set is provided to the matrix: all the names are produced correctly and in a small number of iterations through the BSB algorithm. Figure 13 uses the same matrix, but the input data is now derived from sources whose mean values are shifted about 10 percent, to emulate this parameter shift. 
Figure 12 about here

Figure 13 about here

There were three errors of classification. Emitter 3 was classified as 'Airborn In' instead of 'AA FC'. Emittter 4 was classified as 'SAM target' instead of 'Airborn In'. Emitter 7 was classified as 'Airborn In' rather than the correct 'SAM Target' name. Note that the recalled analog information is also not exactly the correct analog information even for the correctly identified emitters. At a finer scale, the number of iterations required to reach an attractor state was very long. This is a direct measure of the uncertainty of the neural network about the shifted data. Some of the final states were not fully limited, another indication of uncertainty.

Large Classification Data Bases. It would be of interest to see how the system worked with a larger data base. Some information about radar systems is published in Jane's Weapon Systems (Blake, 1988). We can use this data as a starting point to see if a neural network might scale to larger systems. Figure 14 shows the kind of data available from Jane's. Some radars have constant pulse repetition frequency (PRF) and others have highly variable PRF's. (Jane's lists Pulse Repetition Frequency (PRF) in its tables instead of Pulse Repetition Interval (PRI). We have used their term for their data in this simulation.) We represented PRF variability in the state vector coding by increasing the last bar width (Field 7, Figure 15) for highly variable PRF's (see the Swedish radar, for an example.) Also, when a parameter is out of range (the average $P R F$ of the Swedish radar) it is not represented.

Figure 14 about here

Figure 15 about here

We perform the usual partitioning of the state vector into fields, as shown in Figure 15. For this simulation, the frequency scale is so coarse that even enormous changes in frequency would not change the bar coding significantly. We are more interested here in whether the system can handle large amounts of Jane's data. We taught the network 47 different kinds of radar transmitters. Some transmitter names were represented by more than one state vector because they can have several, quite different modes of operation, that is, the parameter part of the code can differ significantly from mode to mode. (The clustering algorithms would almost surely pick 
up different modes as different clusters.) After learning, we provided the measured properties to the the transmitter to see if it could regenerate the name of the country that the radar belonged to. There were only three errors of retrieval from 47 sets of input data, corresponding to 94 percent accurate country identification. This experiment was basically coding a lookup table, using low precision representations of the parameters. Figure 16 shows a sample of the output, with reconstructions of the country, designations, and functions.

Figure 16 about here

Conclusions. We have presented a system using neural networks which is capable of clustering and identifying radar emitters, given as input data large numbers of received radar pulses and with some knowledge of previously characterized emitter types.

Good features of this system are its robustness, its ability to integrate information from co-occurance of many features, and its ability to integrate information from individual data samples.

We might point out that the radar problem is similar to data analysis problems in other areas. For example, it is very similar to a problem in experimental neurophysiology, where action potentials from multiple neurons are recorded with a single electrode. Applications of the neural network techniques described here may not be limited to radar signal processing.

\section{References}

Anderson, J.A. (1972), A simple neural network generating an interactive memory, Mathematical Biosciences, 14, 197-220.

Anderson, J.A. (1983), Neural models for cognitive computation. IEEE Transactions: Systems, Man, and Cybernetics, SMC-13, 799-815.

Anderson, J.A. (1986), Cognitive Capabilities of a Parallel System. In E. Bienenstock, F. Foglemann, and G. Weisbuch. (Eds.) Disordered Systems and Biological organization, Berlin: Springer.

Anderson, J.A. (1987), Concept formation in neural networks: Implications for evolution of cognitive functions. Human Evolution, $\underline{2}, \overline{1} \overline{987}$.

Anderson, J.A. and Mozer, M.C. (1989), Categorization and selective neurons, In: G.E. Hinton and J.A. Anderson (Editors), Parallel Models of Associative Memory (Rev. Ed.), Hillsdale, NJ: Erlbaum.

Anderson, J.A. and Murphy, G.L. (1986), Psychological Concepts in a Parallel system. In J.D. Farmer, A. Lapedes, N. Packard, and B. Wendroff. (Eds.) Evolution, Games, and Learning.. New York: North Holland. 
Anderson, J.A. and Rosenfeld, E., Eds. (1988) Neurocomputing: Foundations of Research, Cambridge, MA: MIT Press.

Anderson, J.A., Silverstein, J.W, Ritz, S.A., and Jones, R.S., Distinctive features, categorical perception, and probability learning: Some applications of a neural model, Psychological Review, 84, 413-451.

Baldi, P. and Hornik, K. (1989), Neural networks and principal component analysis: Learning from examples without local minima. Neural Networks, $2,53$.

Blake, B. (Editor), (1988), Jane's Weapon Systems, (19th Edition), Surrey, U.K.: Jane's Information Group.

Carpenter, G. and Grossberg, S. (1987), ART 2: self organization of stable category recognition codes for analog input patterns, Applied Optics, 26, 4919-4942.

Cottrell, G.W., Munro, P.W. and Zipser, D. (1988). Image compression by back propagation: A demonstration of extensional programming. In N.E. Sharkey (Ed.), Advances in Cognitive Science, Vol. 3. Norwood, NJ: Ablex.

Golden, R.M. (1986), The "Brain state in a box" neural model is a gradient descent algorithm, Journal of Mathematical Psychology, 30, 73-80.

Hinton, G.E. and Anderson, J.A. (Eds., 1989), Parallel Models of Associative Memory (Rev. Ed.), Hillsdale, NJ: Erlbaum.

James, W. (1961/1894), Briefer Psychology, New York: Collier.

Knapp A. and Anderson, J.A. (1984), A theory of categorization based on distributed memory storage. Journal of Experimental Psychology: Learning, Memory and Cognition. 9, 610-622.

Kohonen, T. (1972). Correlation matrix memories, IEEE Transactions on Computers, C-21, 353-359.

Kohonen, T. (1977). Associative Memory. Berlin: Springer. Springer.

Kohonen, T. (1984). Self Organization and Associative Memory. Berlin:

McClelland, J.L. and Rumelhart, D.E., Eds. (1986), Parallel, Distributed Processing, Volume 2, Cambridge, MA: MIT Press.

Miller, G.A. (1956), The magic number seven, plus or minus two: Some limits on our capacity for processing information, Psychological Review, 63, 81-97.

Penz, P.A. (1987), The closeness code, Proceedings of IEEE International Conference on Neural Networks, III-515, IEEE Catalog 87 th0191-7.

Penz, P.A., Katz, A.J., Gately, M.T., Collins, D.R. and Anderson, J.A. (1989), Analog capabilities of the BSB model as applied to the anti-radiation homing missile problem, Proceedings of the International Joint Conference on Neural Nets, II-7.

Rossen, M.L. (1989). Speech Syllable Recognition with a Neural Network, Ph.D. Thesis, Department of Psychology, Brown University, Providence, RI 02912, May, 1989. 
Rumelhart, D.E. and McClelland, J.L., Eds. (1986), Parallel, Distributed Processing, Volume 1, Cambridge, MA: MIT Press.

Rumelhart, D.E. and Zipser, D. (1986), Feature discovery by competitive learning, In D.E. Rumelhart, and J.L. McClelland, Eds., Parallel, Distributed Processing, Volume 1, Cambridge, MA: MIT Press.

Sereno, M.E. (1989)A Neural Network Model of Visual Motion Processing, Ph.D. Thesis, Department of Psychology, Brown University, Providence, RI, May, 1989.

Viscuso, S.R., Anderson, J.A. and Spoehr, K.T., (1989) Representing simple arithmetic in neural networks, In G. Tiberghien, Ed., Advanced Cognitive Sclence: Theory and Applications, London: Horwoods. 


\section{Figures for}

Radar signal Categorization using a Neural Network

James A. Anderson

Department of Cognitive and Linguistic sciences

Box 1978

Brown University, Providence, RI 02912

and

Michael T. Gately, P. Andrew Penz, and Dean R. Collins Central Research Laboratories, Texas Instruments Dallas, Texas 75265 
Figures, Anderson, Gately, Penz and Collins

Caption, Figure 1

Block diagram of the radar clustering and categorizing system.

Caption, Figure 2

Landscape surface of system energy. Several learned examples may contribute to the formation of a single energy minimum which will correspond to a single emitter. This drawing is only for illustrative purposes and is not meant to represent the very high dimensional simulations actually used.

Caption, Figure 3

The Widrow-Hoff procedure learns the error vector. The error vectors early in learning with a small learning constant point toward examples, and the average of the error vectors will point toward the category mean, i.e. ali the examples of a single emitter.

Caption, Figure 4

: Assume an eigenvector is close to a category mean, as will be the result after extensive error correcting, autoassociative learning. The error terms from many learned examples, with a small learning constant, will average to zero and the system attractor structure will not change markedly. (There are very long term 'senility' mechanisms with continued learning, but they are not of practical importance for this application.) 


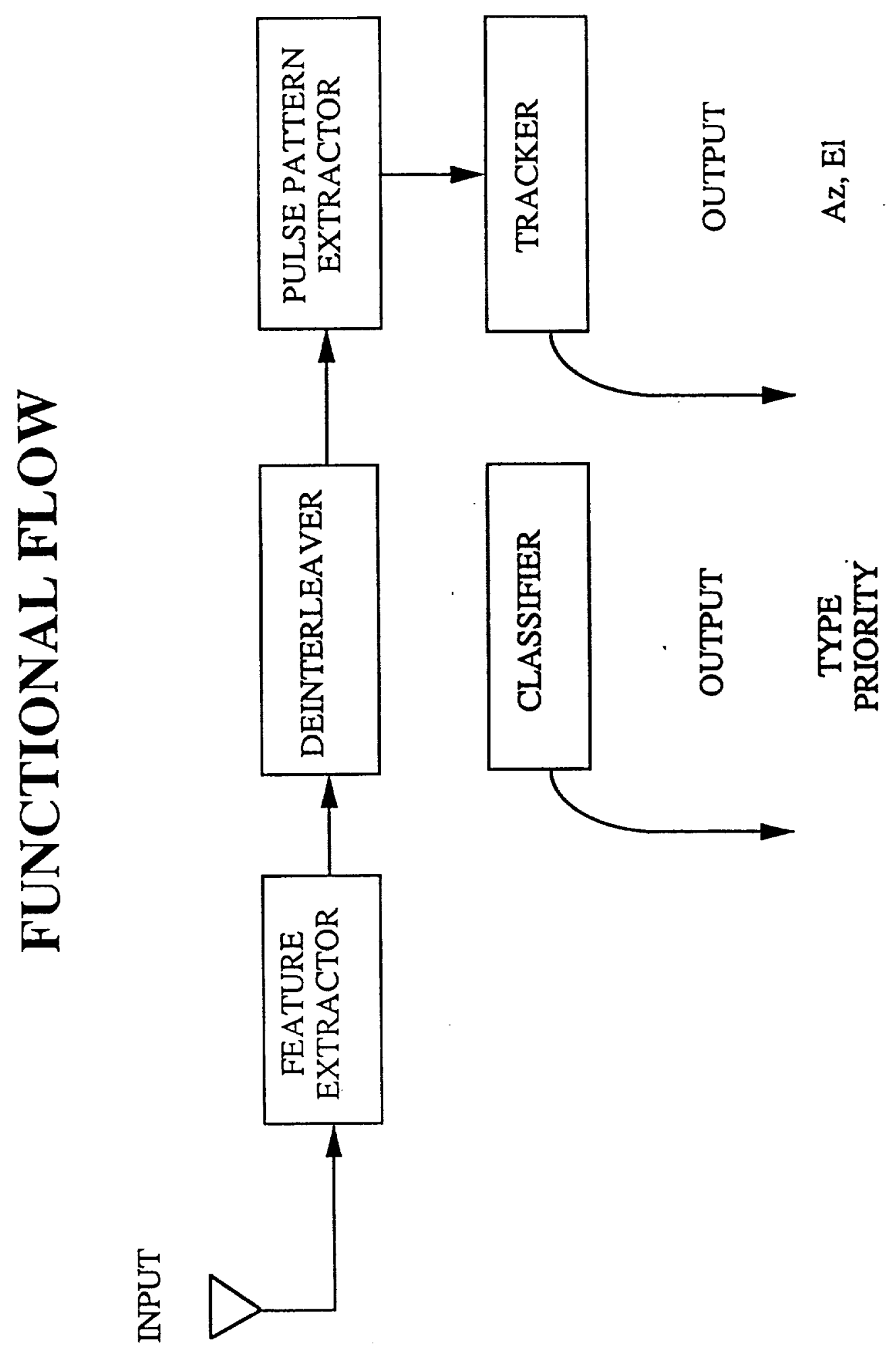




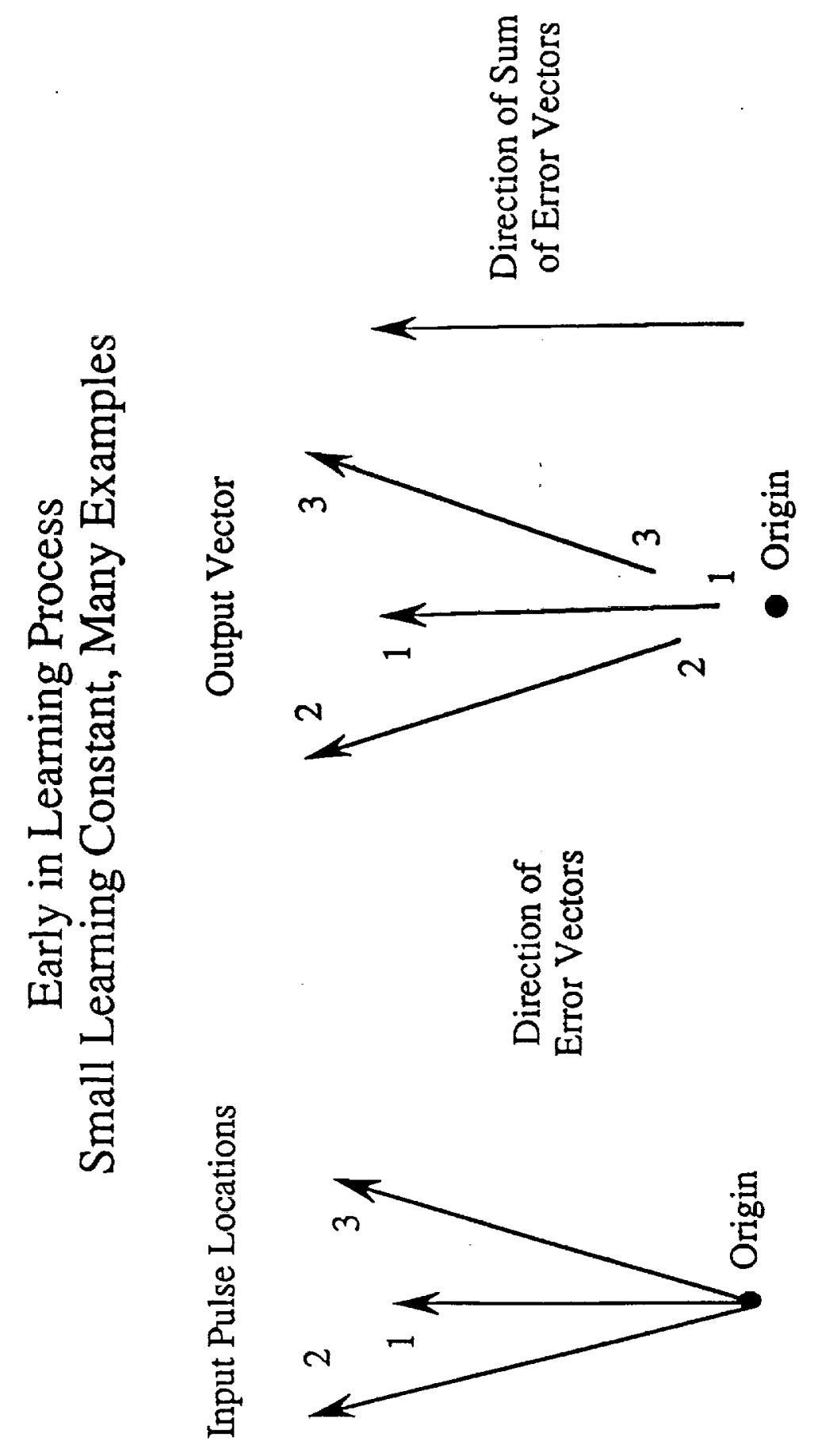




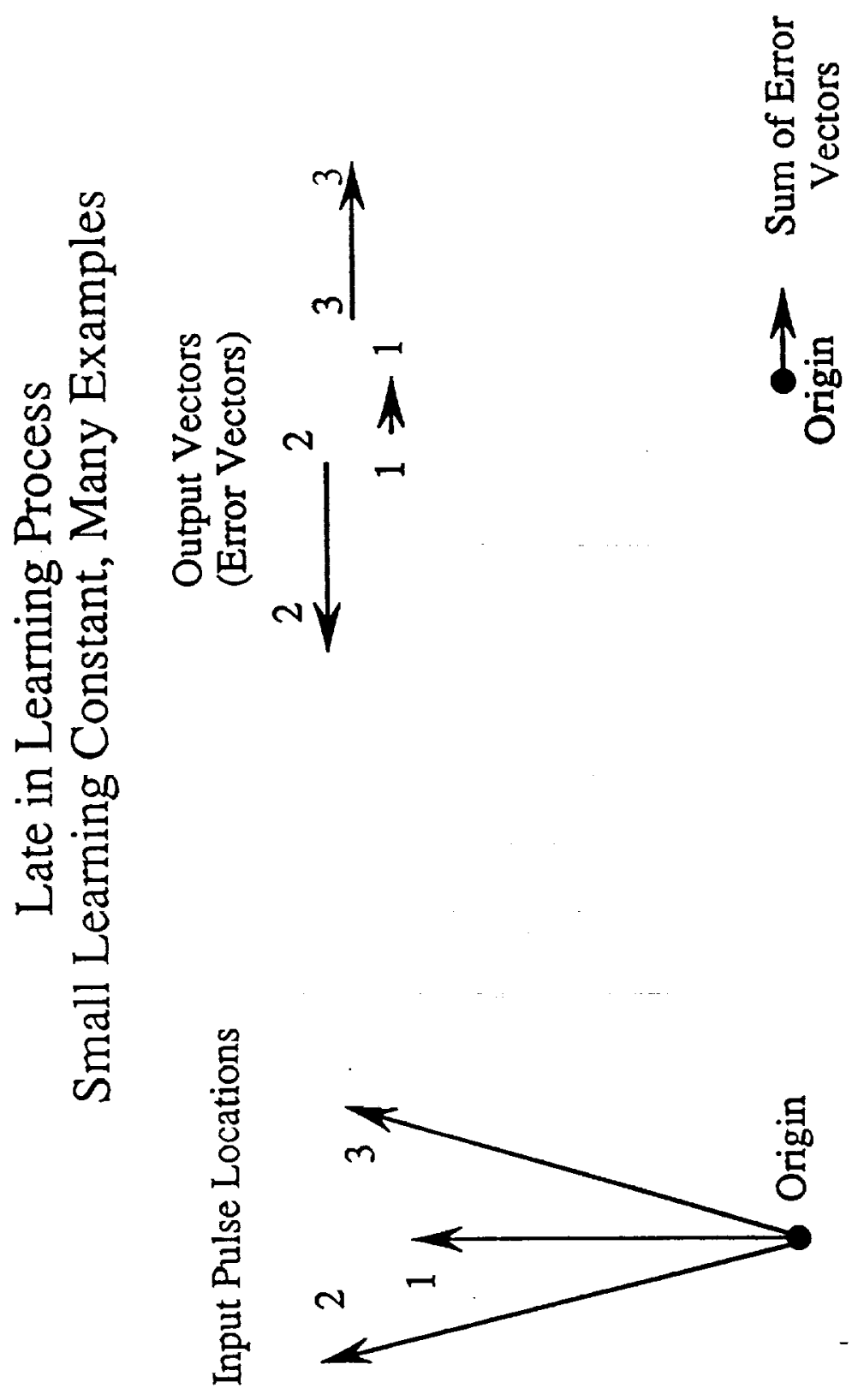




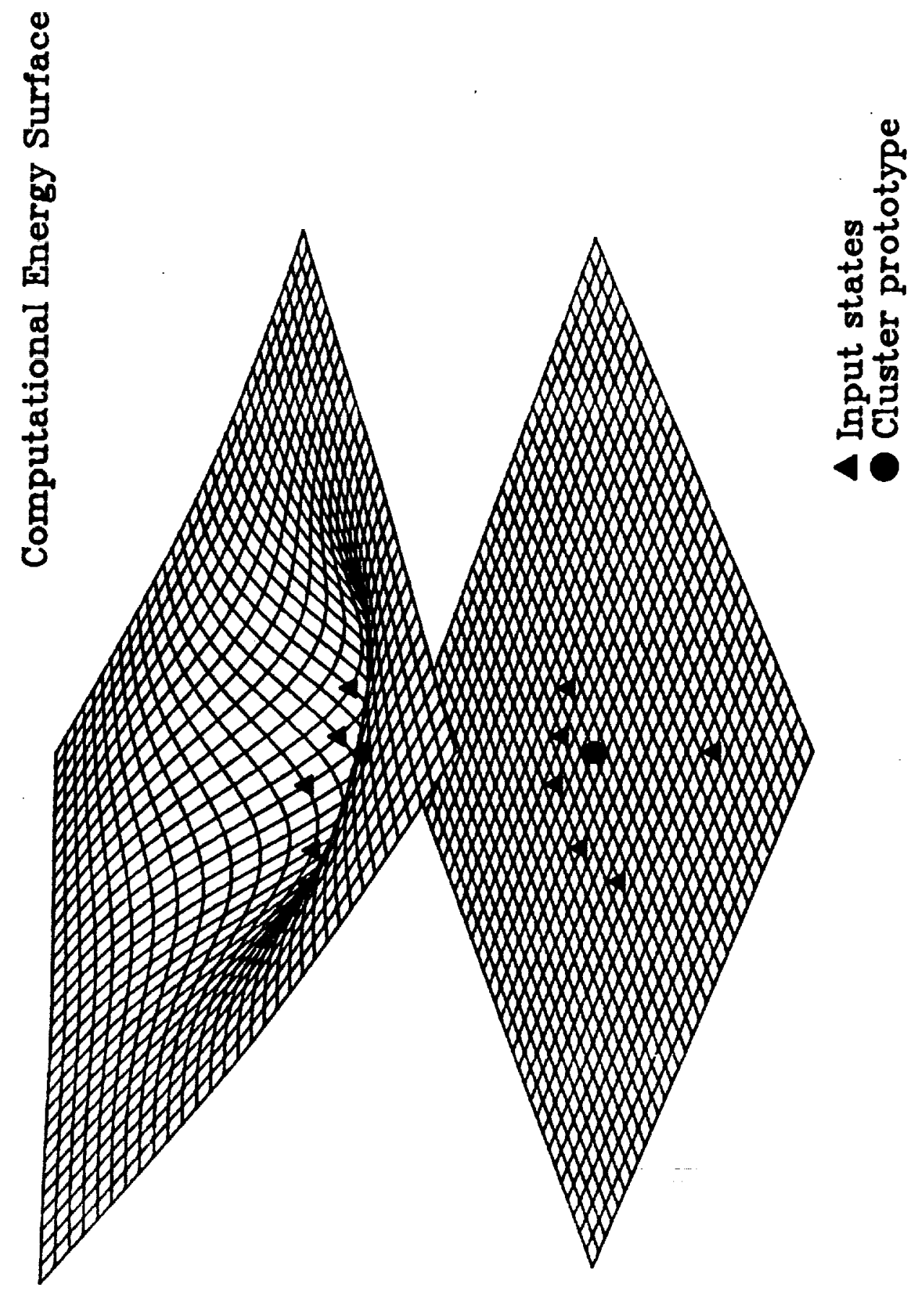


Figures, Anderson, Gately, Penz and Collins

Figure 5

Radar Pulse Fields: Coding of Input Information position of the bar of '=' codes an analog quanitity

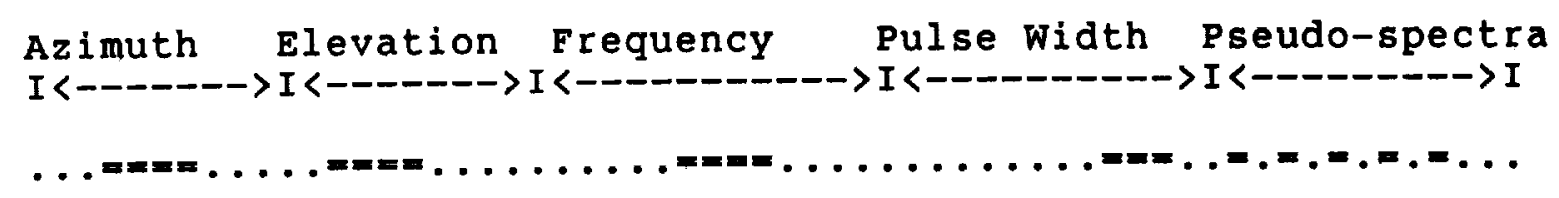

In any field: A move to the left decreases the quantity

A move to the right increases the quantity

Caption, Figure 5

Input representation of analog input data uses bar codes. The state vector is partitioned into fields, corresponding to azimuth, elevation, frequency, pulse width, and a field corresponding to additional information that might become available with advances in receiver technology. 
Figures, Anderson, Gately, Penz and Collins

\section{Figure 6}

\section{clustering by Frequency Information only}

Emitter Final output state

Number

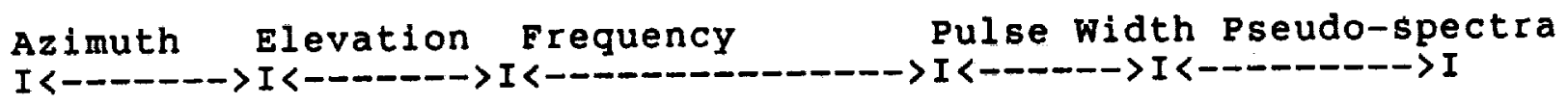

1

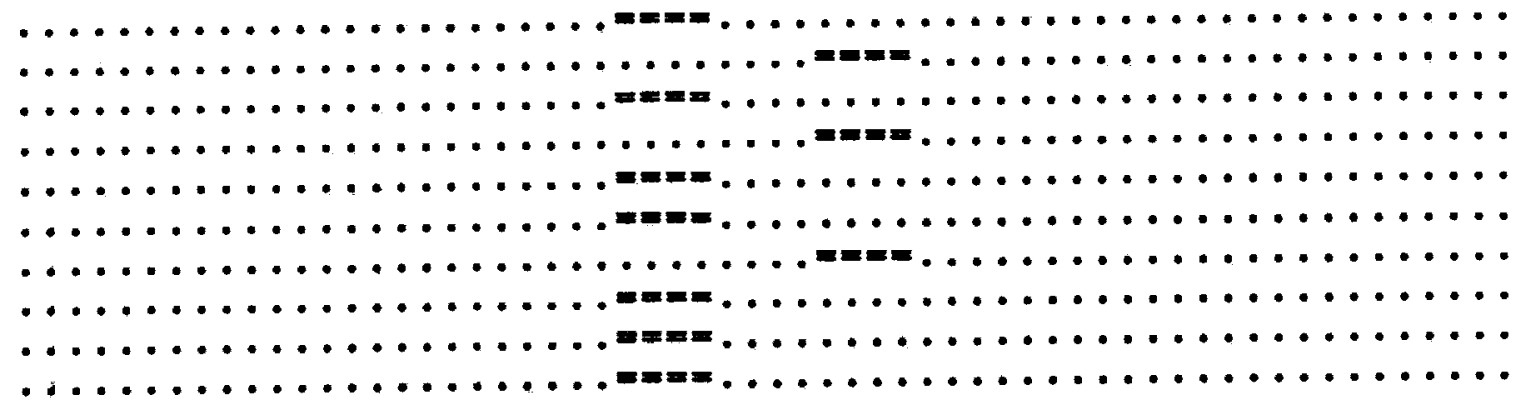

Caption, Figure 6

Final attractor states when only frequency information is learned. Ten different emitter are present, but only two different output states are found. 
Figures, Anderson, Gately, Penz and Collins

Figure 7

Clustering Using Azimuth, Elevation and Frequency Information

Emitter

Final output state

Number
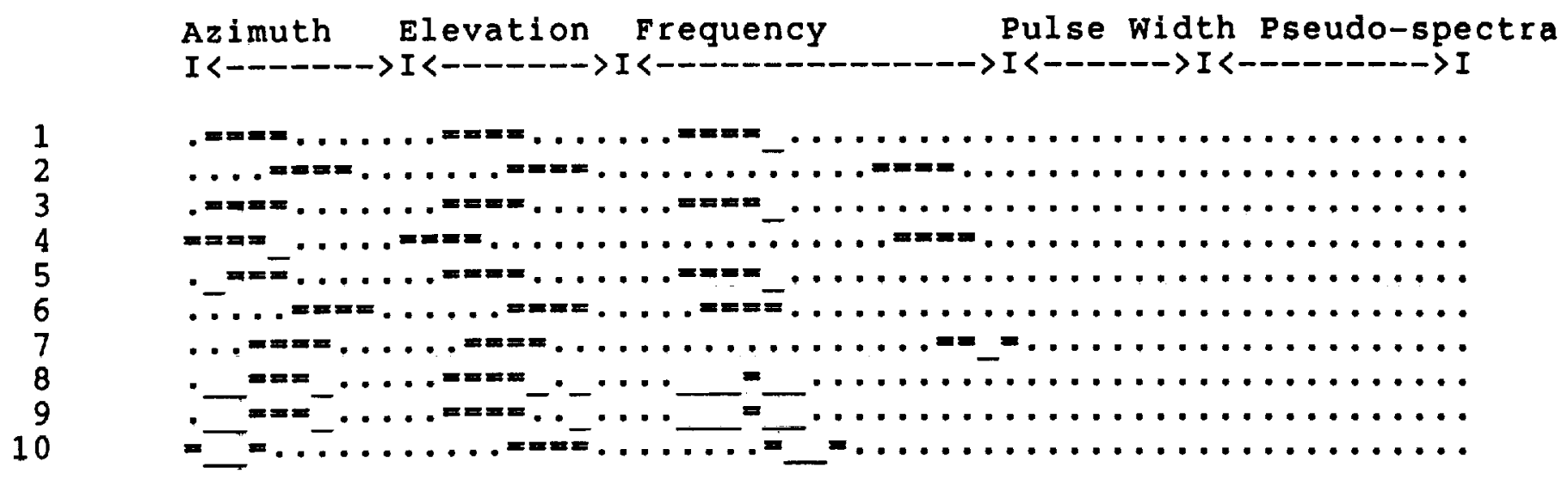

Caption, Figure 7

When azimuth, elevation and frequency are provided for each data point, performance is better. However, two emitters are lumped together, and three others have very close final states. 
Figures, Anderson, Gately, Penz and Collins

\section{Figure 8}
a) $\quad \ldots \ldots=\ldots \ldots$ Monochromatic pulse.
b) $\ldots=.=.=.=.$. subpulses with distinct frequencies. (or some kinds of FM or phase modulation)
c) $\quad,==m==2= \pm=\ldots$ Continuous frequency sweep during the puls

Caption, Figure 8

Suppose we can assume that advances in receiver technology will
allow us to incorporate a crude icartoon of the spectrum of an
individual pulse into the coding of the state vector representing an
example. The spectral information can be included in the state vector
in only slightly processed form.


Figures, Anderson, Gately, Penz and Collins

$$
\text { Figure } 9
$$

Spectrum, Azimuth, Elevation, Frequency

Emitter

Number
Final output state

$$
\begin{aligned}
& \text { Azimuth Elevation Frequency Pulse width Pseudo-spectra }
\end{aligned}
$$

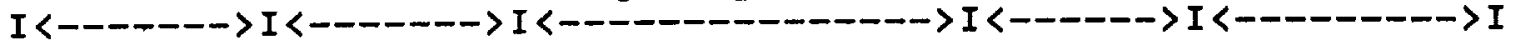

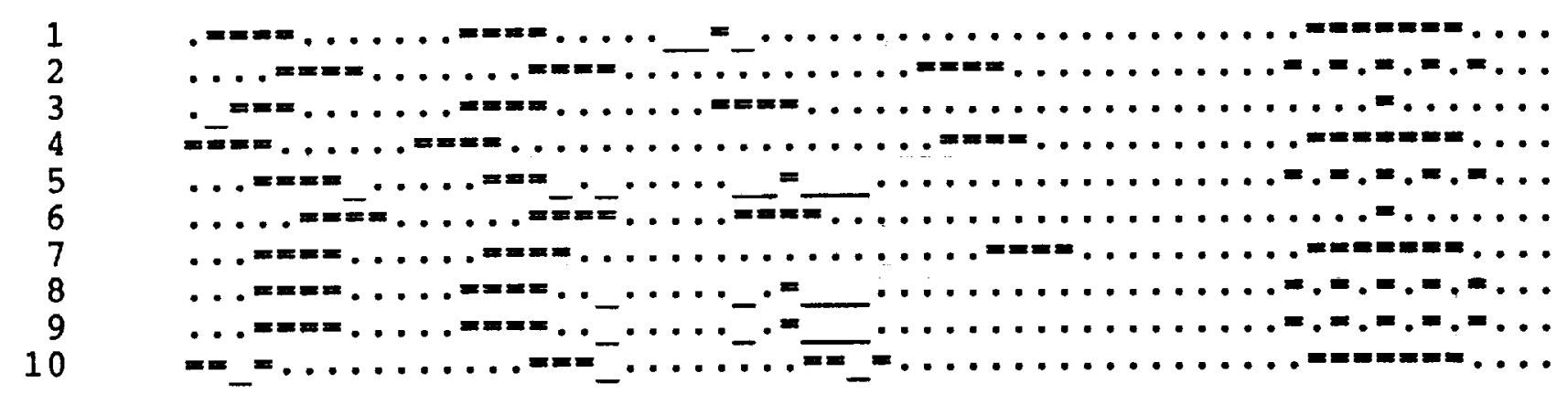

\section{Caption, Figure 9}

Including considerably. well separated. 
Figures, Anderson, Gately, Penz and Collins

Figure 10

Pulse Width, Azimuth, Elevation and Frequency

Emitter Final output state

Number

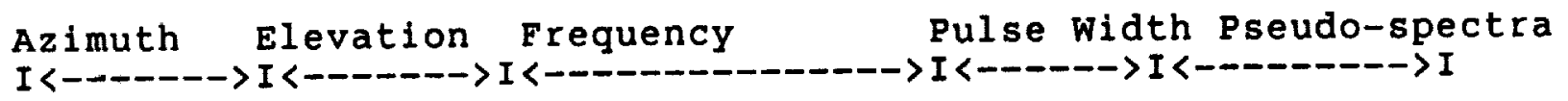

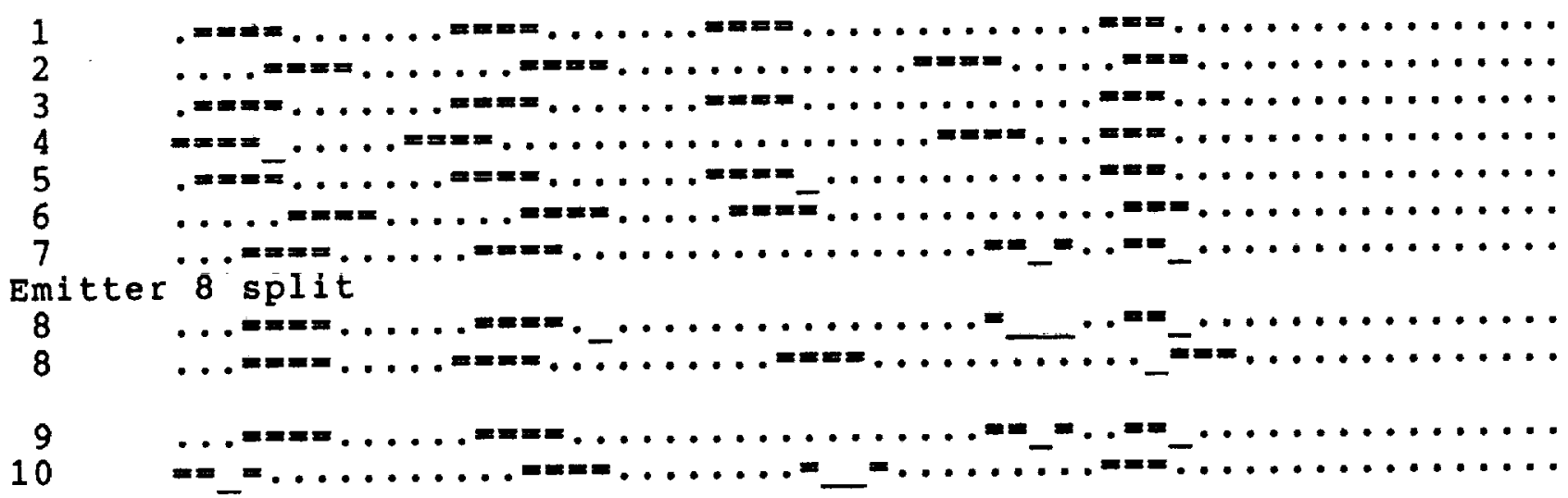

Caption, Figure 10

Suppose we add pulse width information to our other information. close together, a very long pulse width is measured by the receiver circuitry. This gives rise to a bimodal distribution of pulsewidths, and the system splits one category. 
Figures, Anderson, Gately, Penz and Collins

Figure 11

Clustering with All Information

Emitter

Final output state

Number

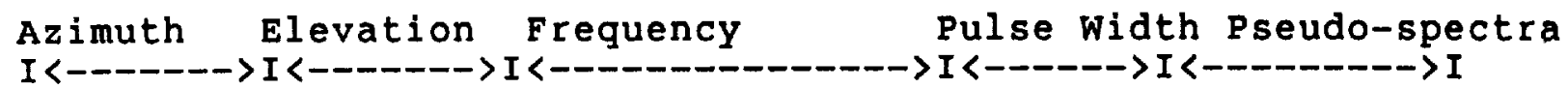

1

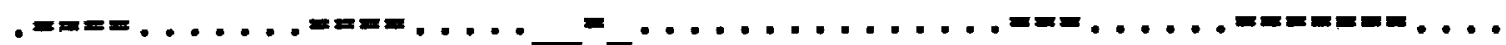

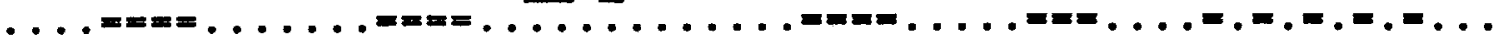

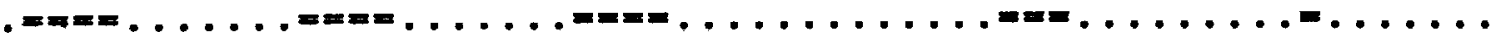

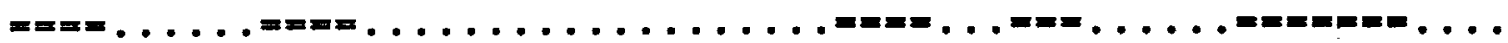

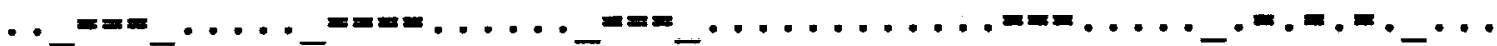

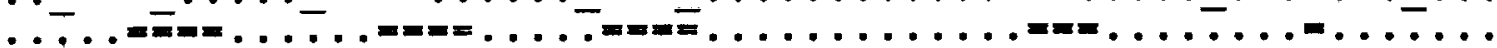

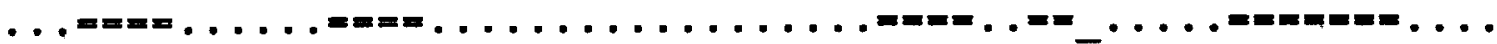

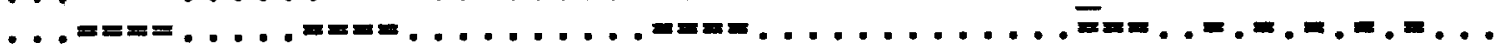

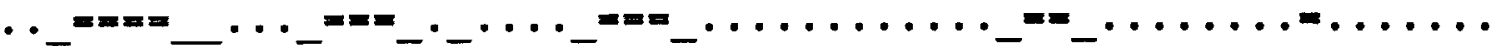

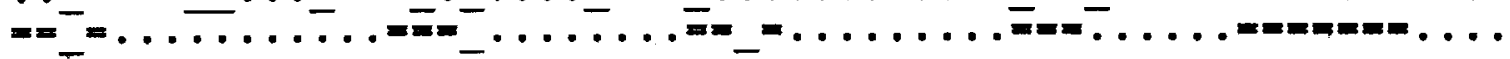

Caption, Figure 11

When all available information is used, ten stable, well separated attractors are formed. This shows that such a network computation can make good use of additional information. 
Figures, Anderson, Gately, Penz and Collins

\section{Figure 12}

Learn normal set, Test normal set

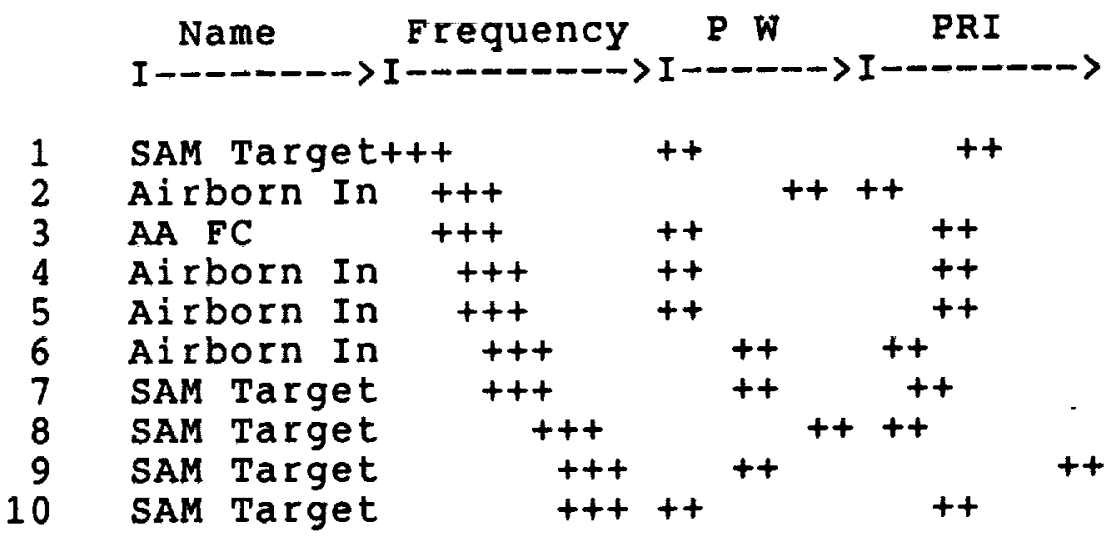

Caption, Figure 12

We can attach identification labels to emitters along with representations of their analog parameters. The names and values used here are random and were chosen arbitrarily. 
Figures, Anderson, Gately, Penz and Collins

Figure 13

Learn Normal set, Test set with shifted Parameters

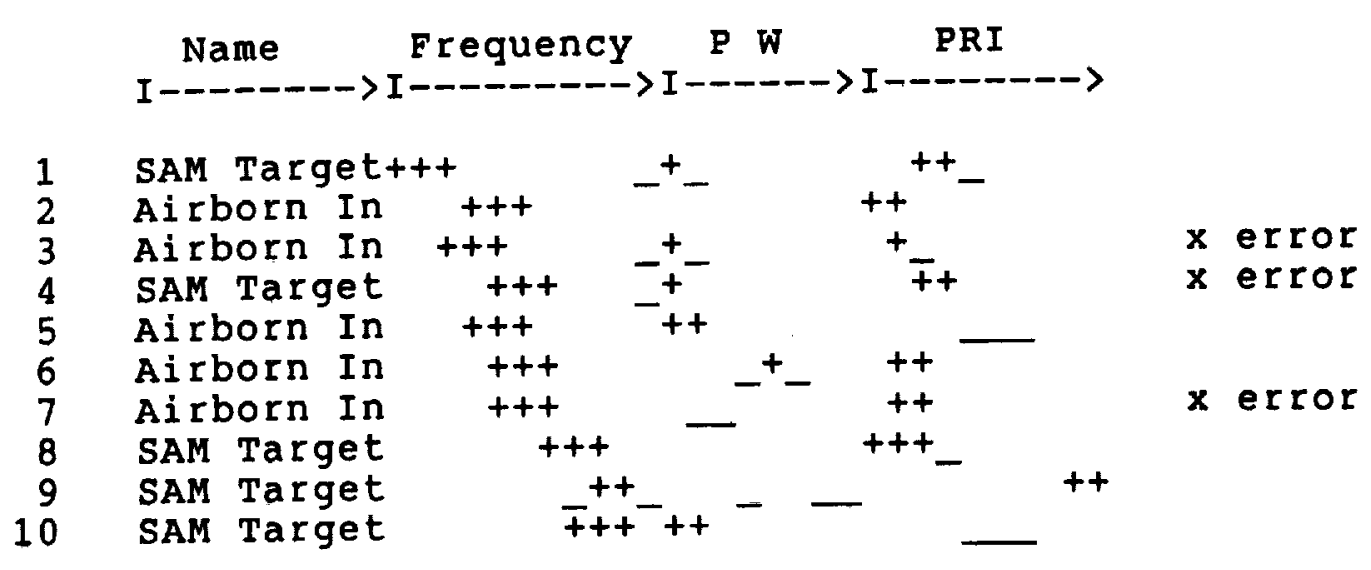

Caption, Figure 13

Even if the emitter parameters shift slightly, it is still possible to make some tentative emitter identifications. Three errors of identification were made. Neural networks are able to generalize to some degree, if the representations are chosen properly. The names and values used here are random and were chosen arbitrarily. 
Figures, Anderson, Gately, Penz and Collins

\section{Figure 14}

\section{Sample Data Obtained from Jane's Weapon Systems}

Three Radars from Jane's:

China, JY-9, search

Frequency : $2.0-3.0 \mathrm{gHz}$

Pulse width : 20 microseconds

PRF : $0.850 \mathrm{kHz}$

PRF Variance: Constant frequency

Sweden, UAR1021, Surveillance

Frequency : $8.6-9.5 \mathrm{gHz}$

Pulse width : $1.5 \mathrm{microseconds}$

PRF : $4.8-8.1 \mathrm{kHz}$

PRF Variance: 3 frequency staggered

USA, APQ113, Firecontrol

Frequency : $16-16.4 \mathrm{gHz}$

Pulse width : $1.1 \mathrm{microseconds}$

PRF : $0.674 \mathrm{kHz}$

PRF Variance: None (Constant frequency)

Caption, Figure 14

Sample data on radar transmitters taken from Jane's Weapon systems. (Blake, 1988). 
Figures, Anderson, Gately, Penz and Collins

Figure 15

Coding into partitioned state Vector:

symbolic Fields:

Continuous Fields:

Field 1 Country

Field 2 Designation

Field 3 Purpose

$\begin{array}{ll}\text { Field } 4 & \text { Frequency } \\ \text { Field } 5 & \text { Pulse width } \\ \text { Field } 6 & \text { PRF } \\ \text { Field } 7 & \text { PRF variation }\end{array}$

Field 4

Field 6

Field 7
PRF Variation
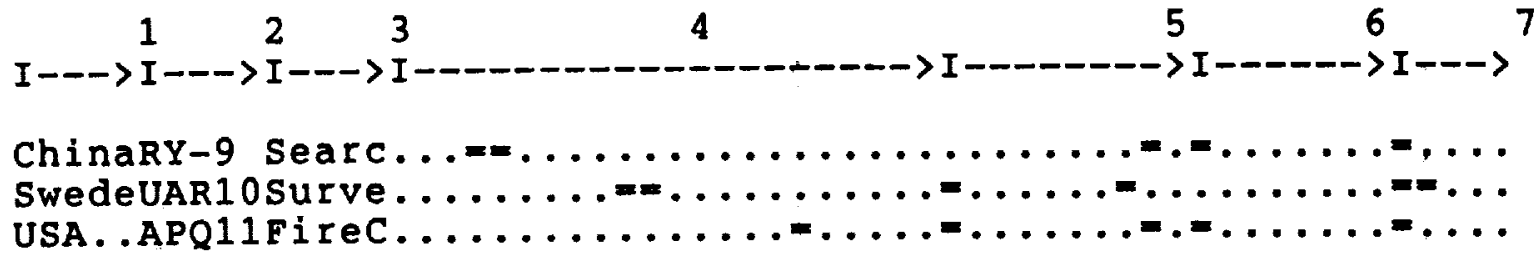

Analog Bar Code Ranges:

Frequency: $\quad 0-14 \mathrm{gHz}$

Pulse width: $0-10 \mathrm{microseconds}$

PRF : $\quad 0-4 \mathrm{kHz}$

PRF Variance: $0-200 \%$ of average PRF

Caption, Figure 15

Bar code representation of Jane's data. Note the presence of both symbolic information such as country name and transmitter designation, and analog, bar coded information such as frequency, pulse width, etc. 
Figures, Anderson, Gately, Penz and Collins

Figure 16

Data Retrieval: Data from Jane's Weapons systems only part of the data

Final output states: 3 errors in reconstructed country

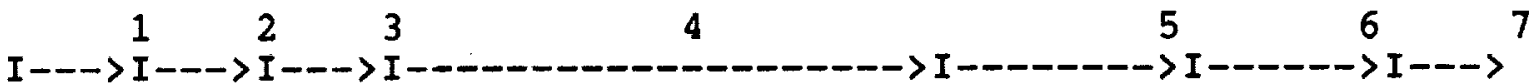

$\mathbf{X}$

ChinarY-9 searc.... ................................

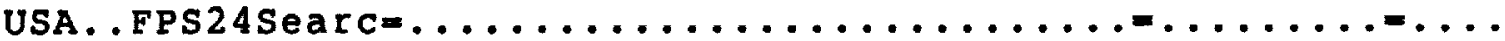

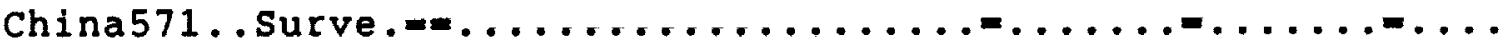

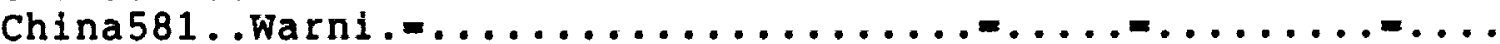

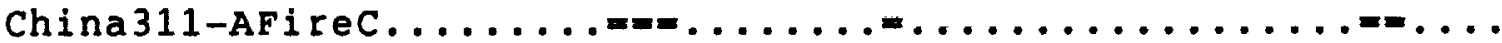
FrancTRS20Surve....................................

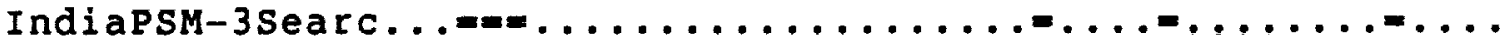
EnglaAs $3-$ FireC . . ...................................

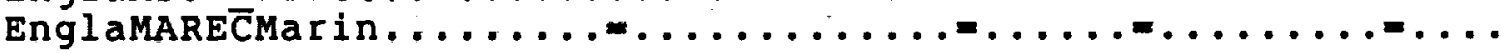
-

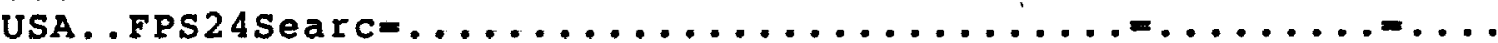

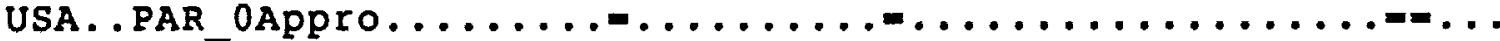

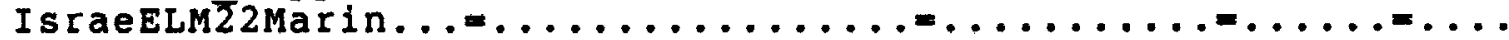
USA. . PR20Appro.................................... . .

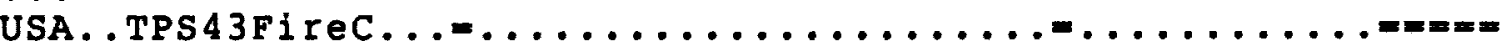

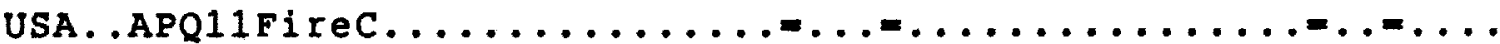

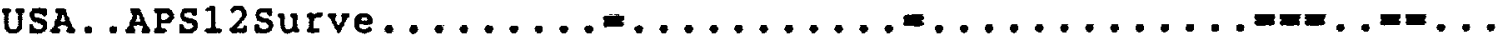

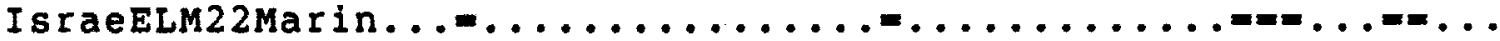

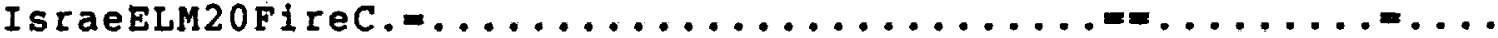

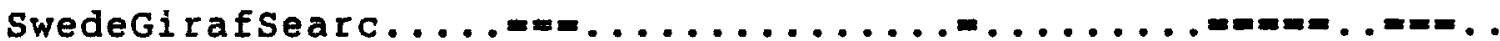

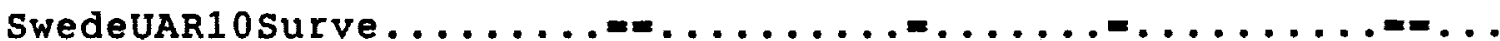

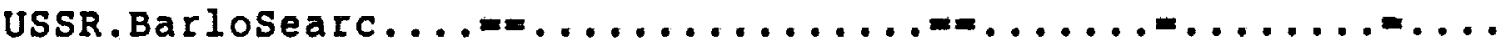

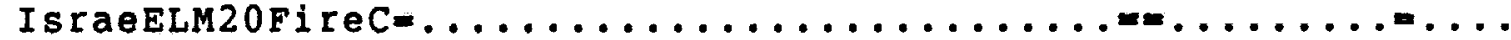

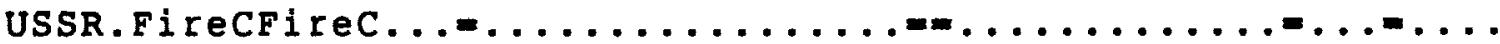

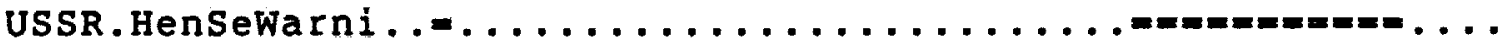

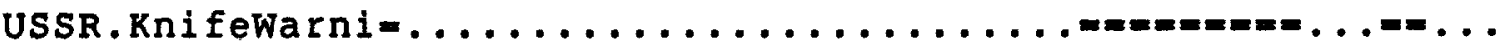
uSSR.JayBiAi rbo ................................m=n=...

Caption, Figure 16

When only analog data is provided at the input, the network will fill in the most appropriate country name. In this trial simulation, a network learned 47 different transmitters and was able to correctly retrieve the associated country in 43 of them. 